\title{
Métodos de reforço microestrutural da hidroxiapatita
}

\section{(Methods of microstructural strengthening of hydroxyapatite)}

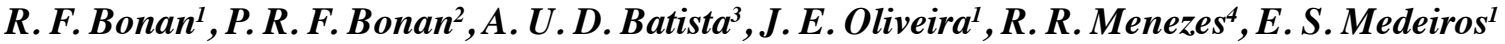 \\ ${ }^{I}$ Departamento de Engenharia de Materiais, ${ }^{2}$ Departamento de Clínica e Odontologia Social \\ Universidade Federal da Paraíba, Campus Universitário, Cidade Universitária, Castelo Branco, J. Pessoa, PB, \\ Brasil 58051-900 \\ ${ }^{3}$ Departamento de Odontologia Restauradora, Universidade Federal da Paraíba, Campus Universitário, \\ Cidade Universitária, Castelo Branco, J. Pessoa, PB, Brasil 58051-900 \\ ${ }^{4}$ Departamento de Engenharia de Materiais, Universidade Federal de Campina Grande, R. Aprígio Veloso 882, \\ Bodocongó, Campina Grande, PB, Brasil 58429-900 \\ robertabonan@gmail.com,pbonan@yahoo.com,andreulisses@yahoo.com.br,juliano.materiais@gmail.com, \\ romualdomenezes@gmail.com,elitonsdemedeiros@gmail.com
}

\begin{abstract}
Resumo
A hidroxiapatita (HAp) é um fosfato de cálcio de fórmula química $\mathrm{Ca}_{10}\left(\mathrm{PO}_{4}\right)_{6}(\mathrm{OH})_{2}$, com relação molar Ca/P 1,67. Este material possui grande importância na tecnologia de biocerâmicas, por ser o constituinte principal da fase mineral de ossos e dentes humanos. Apresenta como algumas de suas aplicações a substituição de tecidos duros danificados, revestimento de próteses ortopédicas e de implantes e reparo de tecidos ósseos. A HAp apresenta resistência mecânica baixa e alta fragilidade o que tornam sua utilização restrita a áreas onde se exige pouco esforço mecânico. Uma das formas de melhorar essas propriedades é associá-la a outros materiais como zircônia, alumina, mulita, titânio e biovidro visando unir as propriedades desejáveis dos dois materiais, bem como pela adição de alguns íons. Este trabalho tem como objetivo descrever as propriedades resultantes da adição de diferentes materiais e íons às propriedades finais da hidroxiapatita. Observou-se que a grande maioria das abordagens de reforço mecânico utilizada atualmente é eficiente na melhoria da resistência à fratura e resistência à flexão e dureza. Verificou-se que apenas o reforço com titânio ainda não tem um efeito claramente elucidado sobre o comportamento mecânico da HAp. A melhora do comportamento mecânico devido à adição do reforço é dependente de fatores como temperatura de sinterização, quantidade de reforço utilizada, ausência de mudanças estruturais indesejáveis, bem como a ausência de outras fases que sejam deletérias às propriedades finais dos materiais produzidos. Palavras-chave: hidroxiapatita, propriedades mecânicas, materiais.
\end{abstract}

\begin{abstract}
Hydroxyapatite (HAp) is a calcium phosphate of chemical formula $\mathrm{Ca}_{10}\left(\mathrm{PO}_{4}\right)_{6}(\mathrm{OH})_{2}$, with a molar ratio of Ca/P equal to 1.67. This material has a great importance in bioceramics technology, being the main mineral phase constituent in human teeth and bones. It is used as a substitute for damaged hard tissues, coating of orthopedic prostheses and implants and bone tissue repair. However, low mechanical strength and high brittleness makes its use restricted to low mechanic strength areas. One way of improving these properties is to combine HAp with other materials such as zirconia, alumina, mullite, titanium, bioglass and ions in order to join desirable properties of both materials. This review aims to describe the properties resulting from the formation of different ions and materials. It was observed that most of the approaches currently used for mechanical reinforcement are effective in improving fracture toughness and flexural strength and hardness.It was also found that the effect of titanium ions on such the mechanical behavior of HAp composite materials has not been completely elucidated. The improvement of the mechanical behavior due to the addition of reinforcement is dependent on factors such as sintering temperature, amount of reinforcement used, absence of undesirable structural changes, as well as presence of other phases that are deleterious to the ultimate properties of produced materials.
\end{abstract}

Keywords: hydroxyapatite, mechanical properties, materials.

\section{INTRODUÇÃO}

Biomateriais podem ser definidos como materiais que são aceitos pelos tecidos vivos, isto é, possuem compatibilidade com sistemas biológicos [1,2]. Podem ser naturais ou sintéticos, adequados principalmente para regeneração total ou parcial de órgãos/tecidos danificados do sistema músculoesquelético [2-4]. As biocerâmicas, amplamente utilizadas na medicina e odontologia, são uma classe importante de biomateriais, que recebem essa denominação por sua origem cerâmica [2]. Possuem propriedades tais como baixa densidade, estabilidade química, alta resistência ao 
desgaste e biocompatibilidade, sendo a última um resultado direto de suas composições que contêm íons normalmente encontrados no ambiente fisiológico, tais como $\mathrm{Ca}^{2+}, \mathrm{K}^{+}$, $\mathrm{Mg}^{2+} \mathrm{e} \mathrm{Na}^{+}$[5-8]. Destacam-se como materiais biocerâmicos hidroxiapatita, alumina, zircônia e biovidros [9]. A Tabela I apresenta algumas das utilizações clínicas das biocerâmicas.

As biocerâmicas são classificadas de acordo com sua interação com os tecidos, como bioinertes ou bioativas, sendo as últimas subdivididas em reabsorvíveis ou não reabsorvíveis [10]. Os materiais bioinertes não formam ligações bioquímicas interfaciais com os tecidos com os quais estão em contato, não apresentando quase nenhuma influência sobre os mesmos, como, por exemplo, a alumina e a zircônia [11]. Já os bioativos possuem essa habilidade de ligação interfacial ao tecido ósseo, como é o caso da hidroxiapatita e dos biovidros [3, 12].

A hidroxiapatita (HAp) é um fosfato de cálcio com fórmula química $\mathrm{Ca}_{10}\left(\mathrm{PO}_{4}\right)_{6}(\mathrm{OH})_{2}$ e razão Ca/P 1,67 $[9,13]$. A estequiometria da HAp possui grande significância, uma vez que leves desequilíbrios na relação $\mathrm{Ca} / \mathrm{P}$ podem levar à formação de outras fases que afetam a interação do material com os tecidos biológicos. Por exemplo, se a relação $\mathrm{Ca} / \mathrm{P}$

Tabela I - Utilizações clínicas das biocerâmicas [8].

[Table I - Clinical uses of bioceramics [8].]

\begin{tabular}{|c|c|}
\hline Aplicação clínica & Materiais \\
\hline Ortopédica & $\begin{array}{c}\text { Alumina } \\
\text { Zircônia estabilizada } \\
\text { Vidros bioativos }\end{array}$ \\
\hline $\begin{array}{l}\text { Preenchimento de } \\
\text { espaços ósseos }\end{array}$ & $\begin{array}{l}\text { Fosfatos de cálcio } \\
\text { Fosfatos tricálcicos }\end{array}$ \\
\hline Implantes dentários & $\begin{array}{c}\text { Alumina } \\
\text { HAp } \\
\text { Vidros bioativos }\end{array}$ \\
\hline $\begin{array}{l}\text { Obliteração de bolsa } \\
\text { periodontal }\end{array}$ & $\begin{array}{l}\text { HAp } \\
\text { Fosfatos de cálcio } \\
\text { Fosfatos tricálcicos } \\
\text { Vidros bioativos }\end{array}$ \\
\hline $\begin{array}{l}\text { Aumento de rebordo } \\
\text { alveolar em ossos } \\
\text { maxilares }\end{array}$ & $\begin{array}{c}\text { HAp } \\
\text { Compósito HA-osso autógeno } \\
\text { Vidros bioativos }\end{array}$ \\
\hline $\begin{array}{l}\text { Reconstrução } \\
\text { maxilofacial }\end{array}$ & $\begin{array}{c}\text { Alumina } \\
\text { HAp } \\
\text { Compósito PE-HAp } \\
\text { Vidros bioativos } \\
\end{array}$ \\
\hline Cirurgia Ortopédica & $\begin{array}{c}\text { Vidros bioativos } \\
\text { HAp }\end{array}$ \\
\hline Otorrinolaringologia & $\begin{array}{c}\text { Alumina } \\
\text { HAp } \\
\text { Vidros bioativos } \\
\text { Compósito PE-HAp }\end{array}$ \\
\hline
\end{tabular}

HAp =hidroxiapatita, $P E=$ polietileno for menor do que 1,67 podem surgir após o processamento fases como o alfa-tricálcio fosfato $(\alpha-\mathrm{TCP})$ ou o betatricálcio fosfato ( $\beta$-TCP); se a relação $\mathrm{Ca} / \mathrm{P}$ for maior do que 1,67, o óxido de cálcio pode estar presente juntamente com a fase HAp [10].

A Fig. 1 apresenta a estrutura cristalina da HAp. A estrutura hexagonal é a fase termodinamicamente mais estável entre os vários tipos de fosfato de cálcio, em $\mathrm{pH}$, temperatura e composição do fluido corporal [14-16], assim como em ar seco ou úmido até $1200^{\circ} \mathrm{C}$ [9].

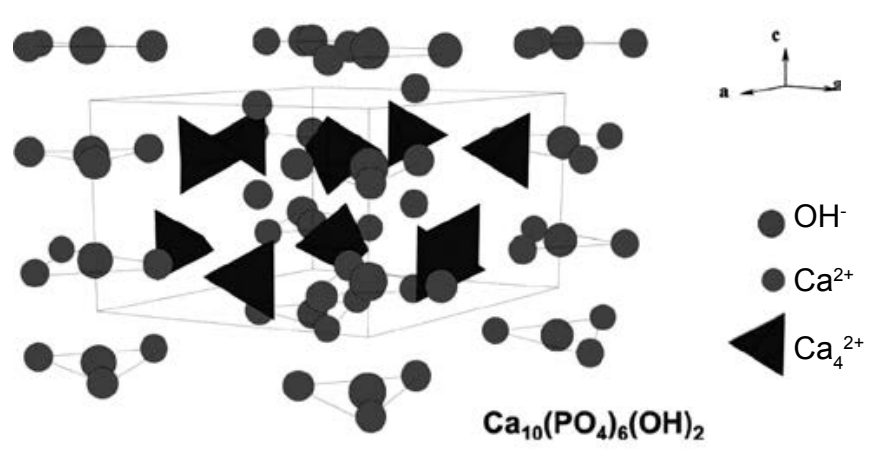

Figura 1: Estrutura cristalina da HAp [6].

[Figure 1: Crystal structure of HAp [6].]

A HAp constitui-se como maior componente do tecido duro humano, constituindo até $69 \%$ da massa óssea natural $[9,10,13,14,17,18]$, além de diferentes proporções dos tecidos dentários, isto é, $96 \%$ do esmalte, $45 \%$ da dentina na forma de apatita carbonatada nanocristalina e $65 \%$ do cemento [19]. Sua obtenção se dá por fontes naturais, tais como ossos bovinos, casca de ovo, conchas e corais [3,14], ou através de métodos químicos, que podem ser classificados em métodos químicos secos, tais como reação de estado sólido e síntese por combustão, e métodos químicos úmidos, como hidrólise, processos hidrotermais, precipitação, síntese sol-gel, entre outros $[9,11,20]$. Pode ser utilizada como material de contato entre componentes artificiais e tecidos humanos [21,22], sendo aplicada na prática clínica na forma granular, densa ou porosa $[4,23,24]$. Tem sua utilização como substituto para tecidos duros danificados [12, 25], de revestimento sobre materiais de implante bioinertes [26,27], utilização em defeitos periodontais [28], reparo de defeitos ósseos, aumento de rebordo alveolar, reconstrução maxilofacial [20] e cirurgias ortopédicas [4].

A HAp apresenta, além da biocompatibilidade, outras propriedades desejáveis como osteocondutividade, que é definida como a habilidade de fornecer a estrutura apropriada para vascularização, infiltração celular, formação de cartilagem e deposição de tecido ósseo [11, 29-31] e propriedades bioativas, devido a sua semelhança de composição química e propriedades cristalográficas com a porção mineral de tecidos duros humanos [16, 32]. Apesar de tais semelhanças químicas, o desempenho mecânico da HAp sintética é muito inferior quando comparado ao tecido ósseo, principalmente quando se refere a sua natureza frágil e baixa tenacidade à fratura e por isso tem utilização clínica ainda 
limitada a regiões não sujeitas a grandes esforços mecânicos [20, 33-35].Visando melhorar essas propriedades, através do controle da densidade e microestrutura, vários métodos de reforço microestrutural da HAp têm sido utilizados, principalmente através da formação de compósitos $[5,23$, 31, 33, 36-42].

Com o intuito de explorar as possibilidades de reforço microestrutural da HAp, o presente trabalho tem como objetivo descrever alguns desses métodos, destacando suas principais vantagens e desvantagens.

\section{REFORÇO MICROESTRUTURAL E HAp}

Durante os últimos cinqüenta anos, os avanços nos estudos de biocerâmicas têm tido significativa contribuição para o desenvolvimento da indústria moderna de saúde, melhorando a qualidade da vida humana [7]. Como resultado, existe um impulso para o desenvolvimento de materiais substitutos ósseos que não danificam o tecido saudável, não representam risco de contaminação viral ou bacteriana dos pacientes, e podem ser fornecidos independente do momento e quantidade [8].

As biocerâmicas para serem utilizadas em tecidos vivos, além de sua compatibilidade com o meio biológico, devem possuir capacidade de suportar tensões complexas no local da aplicação [7]. Como as aplicações clínicas das biocerâmicas estão em progresso, suas limitadas propriedades mecânicas estão se tornando aparentes, tendo seu uso prejudicado em áreas sujeitas a altas cargas, tais como nos reparos de defeitos ósseos do fêmur e da tíbia. Por conseguinte, o desenvolvimento de materiais bioativos com propriedades mecânicas melhoradas tem sido proposto [5, 24, 37, 42].

Dentre as cerâmicas bioativas utilizadas, destaca-se HAp e um dos meios utilizados para melhorar suas propriedades mecânicas, principalmente tenacidade à fratura e dureza, tem sido a elaboração de compósitos. Com isso, tem-se a conseqüente ampliação das indicações para utilização da mesma [25, 31, 33, 36-41]. Como materiais utilizados como agente de reforço para a HAp destacam-se a utilização da zircônia [43-45], alumina [3, 39, 46], mulita [47-49], titânio $[36,50,51]$ e biovidro $[13,42,52]$. Outra abordagem que vem sendo explorada é através da adição de íons em solução sólida na HAp [53-63].

\section{Zircônia}

A zircônia é um material que tem sido comumente usado visando unir suas excelentes propriedades mecânicas tais como elevada tenacidade à fratura, dureza, resistência ao desgaste, inércia química e baixa condutividade térmica à bioatividade da HAp [12, 22, 43-45, 64, 65]. Todavia, a união entre zircônia e HAp apresenta algumas desvantagens devido à decomposição da HAp em outras fases, bem como as possíveis transformações alotrópicas da zircônia da fase tetragonal para a cúbica durante a sinterização, resultando na redução das propriedades mecânicas e biocompatibilidade do compósito [15, 37-40, 65]. A razão da transformação de fase na zircônia bem como da decomposição da HAp está atribuída à difusão do íon $\mathrm{Ca}^{2+}$ da HAp para a zircônia $[15,66]$. A presença da zircônia diminui a temperatura de decomposição da HAp e esse processo resulta na mudança das propriedades físicas e químicas do compósito, afetando diretamente seu desempenho final [67]. A quantidade formada de outras fases reabsorvíveis, tais como o $\mathrm{CaZrO}_{3}$, $\alpha$-TCPe $\beta$-TCP, ocorre a partir de $950^{\circ} \mathrm{C}$, tendo seu aumento diretamente relacionado ao aumento da temperatura de sinterização [37, 40, 65]. Por outro lado, a utilização de temperaturas de sinterização menores, bem como a aplicação de tempos de processamento menores, resulta em um grau mais baixo de densificação, quando se utiliza métodos convencionais de sinterização $[37,65]$.

Com o objetivo de reduzir o processo de decomposição da HAp são utilizados processos de sinterização específicos que se utilizam de ciclos de queima rápida e/ou de menores temperaturas de sinterização, tais como a prensagem a quente, prensagem isostática a quente e sinterização por plasma (spark plasma sintering - SPS). A vantagem da utilização desses processos é a obtenção de alta densificação

Tabela II - Propriedades mecânicas da zircônia parcialmente estabilizada comparadas a diferentes tecidos humanos e outros biomateriais [43].

[Table II - Mechanical properties of partially stabilized zircon (PSZ) compared with different human tissues and biomaterials [43].]

\begin{tabular}{lcccc}
\hline \multicolumn{1}{c}{ Material } & $\begin{array}{c}\text { Resistência à } \\
\text { compressão } \\
(\mathrm{MPa})\end{array}$ & $\begin{array}{c}\text { Módulo de } \\
\text { Young } \\
(\mathrm{GPa})\end{array}$ & $\begin{array}{c}\text { Módulo de } \\
\text { resiliência } \\
(\mathrm{MPa})\end{array}$ & $\begin{array}{c}\text { Dureza } \\
\text { Micro-Vickers }\end{array}$ \\
\hline Osso humano cortical & $88-230$ & $3-30$ & - & - \\
Dente humano (dentina) & $300-380$ & $15-20$ & 62,7 & - \\
Dente humano (esmalte) & $250-550$ & $10-90$ & - & 340 \\
HAp & $300-900$ & $80-120$ & $6-13$ & $450-530$ \\
$\beta$ - TCP & $450-650$ & $90-120$ & - & \\
PSZ & $1700-2000$ & $195-210$ & $30-500$ & $1100-1200$ \\
\hline$\beta$ - TCP: fosfato tricálcio & & & & \\
PSZ: zirconia parcialmente estabilizada & &
\end{tabular}


do compósito, ao mesmo tempo em que minimiza a tendência de reação e decomposição dos constituintes do compósito em altas temperaturas $[15,37]$.

Da mesma forma, com oobjetivo de evitar a transformação de fase da zircônia, aditivos tais como os óxidos de cálcio $(\mathrm{CaO})$, magnésio $(\mathrm{MgO})$ e de ítrio $\left(\mathrm{Y}_{2} \mathrm{O}_{3}\right)$ são utilizados misturados à zircônia pura para gerar um material multifásico chamado de zircônia parcialmente estabilizada (PSZ), cuja microestrutura na temperatura ambiente consiste na zircônia cúbica como fase majoritária com precipitados de zircônia monoclínica e tetragonal como fases minoritárias [43-45, 66, 67]. A Tabela II mostra algumas propriedades mecânicas da zircônia parcialmente estabilizada comparadas a diferentes tecidos humanos e outros biomateriais.

A zircônia estabilizada com óxido de ítrio é usada para aplicações biomédicas, devido sua elevada tenacidade [68]. Possui estrutura cristalina que varia de um sistema multifásico monoclínico e tetragonal para uma simetria cúbica com o aumento de conteúdo de $3 \mathrm{~mol} \% \mathrm{Y}_{2} \mathrm{O}_{3}$ até $8 \mathrm{~mol} \%$ [67]. A zircônia parcialmente estabilizada por cálcio tem sido amplamente estudada devido a sua elevada resistência e tenacidade, além de ser um material bioinerte. Sua adição na matriz de HAp leva a um aumento das propriedades mecânicas do compósito, sem afetar sua biocompatibilidade [43-45, 68].

\section{Alumina}

A alumina também tem sido amplamente investigada como um agente de reforço para HAp e sua utilização visa principalmente a melhoria da resistência mecânica da matriz [3, 39, 46, 69-72]. Porém, uma limitação desse compósito é a grande diferença de coeficiente de expansão térmica entre os dois materiais [72]. Verifica-se também a degradação da HAp na presença da alumina, com a formação de fosfato de tricálcio e aluminatos de cálcio durante a sinterização e consolidação do corpo [46]. Referente a essa questão, o efeito da adição de $20 \%$ em vol de alumina com partículas menores do que $1 \mu \mathrm{m}$ em matriz de HAp por prensagem a quente (uniaxial), em $1200{ }^{\circ} \mathrm{C}$, foi avaliado [72], sendo observada uma melhoria de propriedades como a resistência à flexão e tenacidade à fratura de cerca de $100 \mathrm{MPa} \mathrm{e}$ $0,7 \mathrm{MPa} \cdot \mathrm{m}^{1 / 2}$ respectivamente para $200 \mathrm{MPa}$ e $1,5 \mathrm{MPa}$. $\mathrm{m}^{1 / 2}$. Temperaturas de sinterização maiores que $1200{ }^{\circ} \mathrm{C}$ provocaram efeitos não desejáveis devido à decomposição da HAp em â- TCP. Já a adição dessa mesma proporção de alumina, com partículas de tamanho médio de $0,3 \mu \mathrm{m}$, na matriz de HAp (previamente calcinada a $900{ }^{\circ} \mathrm{C}$ por $3 \mathrm{~h}$ ) com sinterização na temperatura de $1400{ }^{\circ} \mathrm{C}$ por 2 ou $4 \mathrm{~h}$, evidenciou que, como resultado do processo de calcinação, ocorreu a decomposição parcial da hidroxiapatita, com o aparecimento de fases tais como $\alpha-\mathrm{Ca}_{3}\left(\mathrm{PO}_{4}\right)_{2}$, além de fases de aluminatos de cálcio, que podem reduzir a resposta biológica do compósito. Isto ocorre porque o fosfato de cálcio $\alpha-\mathrm{Ca}_{3}\left(\mathrm{PO}_{4}\right)_{2}$ possui resposta biológica diferente da HAp original e os aluminatos de cálcio possuem solubilidades desconhecidas e reações teciduais após implantação [46].
Por outro lado, altas temperaturas de sinterização são necessárias para que se possa obter a densificação. Além da decomposição da HAp, foi observada a presença de microtrincas na interface entre a fase de fosfato de cálcio e fase de aluminato de cálcio e também no contorno de grão na fase de fosfato de cálcio. Essas microtrincas provavelmente resultaram das transformações de fase, evidenciando que o uso de altas temperaturas e tamanhos mais finos de partículas acentuaram a reatividade entre as fases e a degradação do sistema [46]. Curiosamente, quando um composto com flúor, como o $\mathrm{MgF}_{2}$ ou o $\mathrm{CaF}_{2}$, foi adicionado ao compósito de HAp-alumina (20 vol.\%) ocorreu a completa inibição da decomposição da HAp até temperaturas abaixo de $1400^{\circ} \mathrm{C}$ devido à estabilização da estrutura cristalina da mesma [73].

Já a sinterização através da técnica de prensagem isostática a quente a $1250{ }^{\circ} \mathrm{C}$ por $2 \mathrm{~h}$ permitiu a densificação do compósito alumina-HAp acima de 99,5\%, sem causar a degradação da HAp, permitindo ainda a melhoria da resistência à flexão do compósito, que teve relação direta com o aumento da quantidade de alumina; esse efeito foi evidente quando a quantidade do reforço foi de $30 \mathrm{vol} . \%$, onde o aumento foi de duas vezes no valor da resistência quando comparada a da HAp pura [74], conforme está mostrado na Fig. 2.

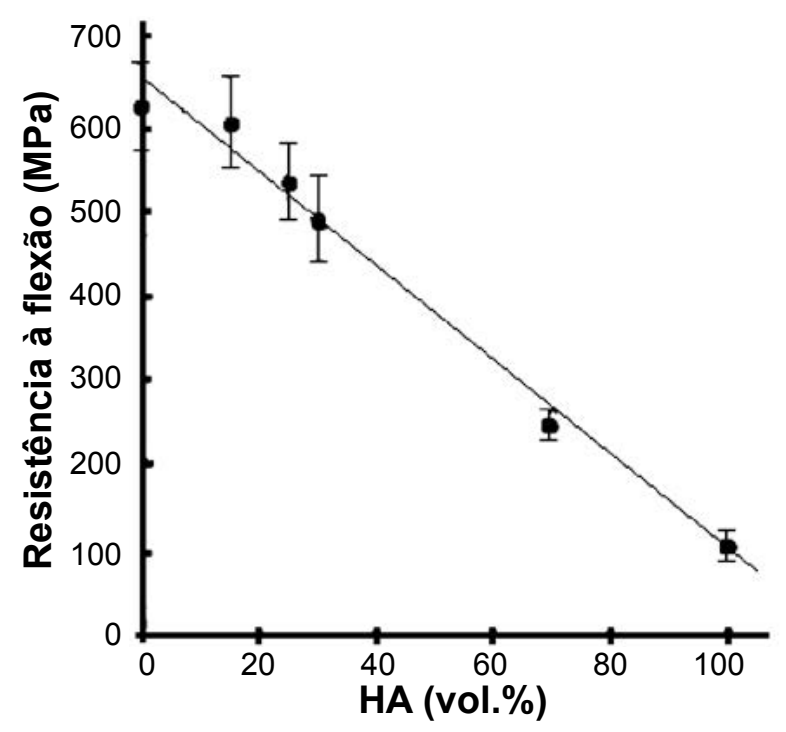

Figura 2: Resistência à flexão de compósitos $\mathrm{HAp} / \mathrm{Al}_{2} \mathrm{O}_{3}$ em função do conteúdo de hidroxiapatita [74].

[Figure 2: Bending strength of composites $\mathrm{HAp} / \mathrm{Al}_{2} \mathrm{O}_{3}$ as a function of hydroxyapatite content [74].]

$\mathrm{O}$ uso de partículas de alumina com formato lamelar (platelets) como reforço para a matriz de HAp também vem sendo investigado. Sua adição pode melhorar propriedades mecânicas como a tenacidade à fratura e dureza, porém, dependendo da rota de processamento utilizada, existe uma tendência de formação de aglomerados no compósito, o que limita esse processo de melhora do material. É importante evitar a presença de tais aglomerados, para prevenir ou reduzir a formação de defeitos microestruturais no compósito [69-71, 75]. Além disso, os mecanismos de 
melhoria de resistência e dureza da matriz de HAp com a adição de partículas de alumina com formato lamelar estão diretamente relacionados ao tamanho de grão da HAp, ao tamanho, forma e volume dessas partículas. A diferença de expansão térmica entre a HAp e a alumina e a geometria anisotrópica das partículas do reforço podem levar à presença de microtrincas no compósito e, por conseguinte, à deterioração do efeito reforçante destas [70]. A adição de 20 vol.\% de partículas com formato lamelar de $\mathrm{Al}_{2} \mathrm{O}_{3}$ na matriz de HAp, com posterior sinterização por prensagem a quente em $1200{ }^{\circ} \mathrm{C}$ por $30 \mathrm{~min}$, gerou um aumento na dureza em $65 \%$, em relação à HAp pura, sem causar a degradação de fases. Já a tenacidade à fratura do compósito, em comparação com a HAp pura, foi 3 a 4 vezes superior com a adição de 20 vol.\% de $\mathrm{Al}_{2} \mathrm{O}_{3}$ [69].

A utilização da alumina em conjunto com a zircônia como reforço da matriz de HAp também vem sendo estudada [25, 39]. O sistema zircônia-alumina é conhecido por sua alta resistência à flexão, sendo classificada como uma cerâmica bioinerte [38]. Quando o efeito da adição de 15 e 30 massa\% de $\mathrm{ZrO}_{2}-\mathrm{Al}_{2} \mathrm{O}_{3}$ à matriz de $\mathrm{HAp}$ foi avaliado, houve melhoria significativa da resistência à flexão, mas não houve alteração da tenacidade à fratura, quando foi adicionado 15 massa\% do reforço [25]. Quando a adição foi de 30 massa\%, tanto a resistência à flexão quanto a tenacidade à fratura diminuíram em todas as temperaturas de sinterização utilizadas, o que foi atribuído à formação em grande quantidade das fases $\mathrm{TCP}$ e $\mathrm{ZrO}_{2}$ cúbica [25].

A resistência à flexão e a tenacidade à fratura da HAp podem ser melhoradas de forma significativa pela adição de diferentes proporções de pó de zircônia tetragonal recoberta com alumina. $\mathrm{O}$ recobrimento foi efetivo na redução das reações deletérias entre a HAp e a zircônia. A adição de 15 vol.\% de zircônia e 30 vol. \% de alumina à HAp pelo método de revestimento gerou um aumento de cerca de 3 vezes

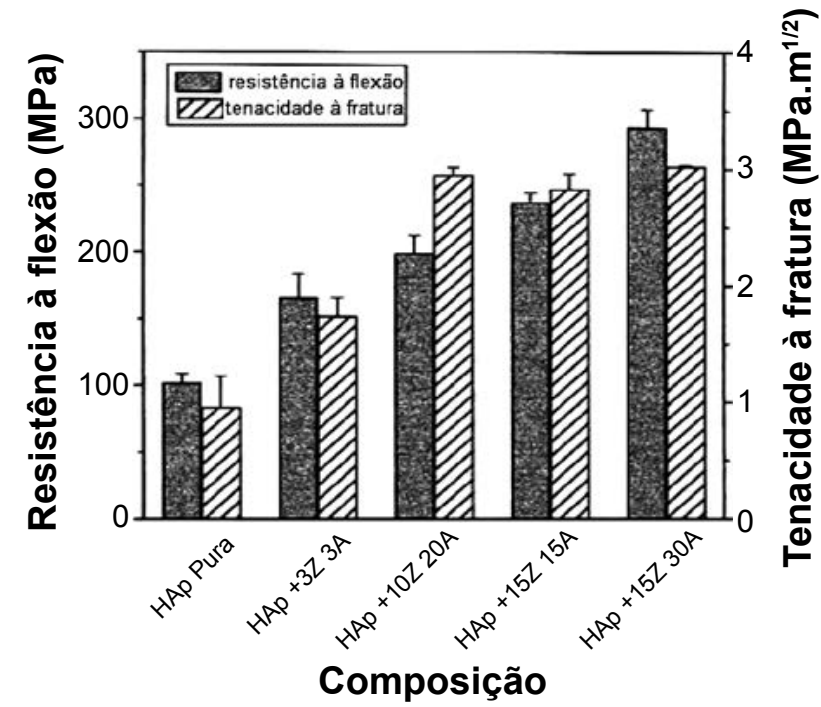

Figura 3: Resistência à flexão e tenacidade à fratura da HAp contendo várias quantidades de zircônia revestida com alumina [39].

[Figure 3: Flexural strength and fracture toughness of the HAp containing various amounts of zirconia coated with alumina [39].] tanto na resistência à flexão como na tenacidade à fratura do compósito em relação à HAp pura, conforme mostrado na Fig. 3. A preservação das partículas de zircônia em sua fase tetragonal na matriz de HAp foi relacionada à melhoria das propriedades mecânicas [39].

\section{Titânio}

Outro material também utilizado como reforço para a estrutura da HAp é o titânio. Ele é usado para formar compósitos que são usados principalmente como revestimento de implantes, visando unir as propriedades mecânicas do titânio com as propriedades biológicas da HAp [35, 36, 50, 51, 76-78]. O titânio além de melhorar as propriedades mecânicas da HAp, ainda possui outra característica muito favorável que é sua biocompatibidade, isto é, possui capacidade de aumentar adesão de osteoblastos e de induzir crescimento celular [36, 78]. Para a produção desses revestimentos são usadas técnicas tais como: "ionsputtering", deposição eletroquímica e "spray plasma" que utilizam altas temperaturas, tendo como consequiência mudanças estruturais e processos de decomposição, ocorrendo a presença de outras fases, tais como o dióxido de titânio $\left(\mathrm{TiO}_{2}\right)$ e a perovskita $\left(\mathrm{CaTiO}_{3}\right)$ [36, 77, 78]. Mesmo em técnicas que utilizam menores temperaturas no processamento, ainda houve a presença de outras fases como o $\mathrm{TiO}_{2}$ [77]. Para que haja a alta densificação do compósito, em geral são necessárias temperaturas de sinterização em torno de $1300{ }^{\circ} \mathrm{C}$, o que também favorece os processos de decomposição da HAp, enquanto temperaturas de sinterização em torno de $1000{ }^{\circ} \mathrm{C}$ geram uma pobre densificação do sistema [36], o que evidencia, novamente, a dificuldade em se obter compósitos contendo HAp que possuam elevada densificação, sem que haja alteração na estabilidade das fases presentes.

A reação química entre os componentes, titânio e HAp, também é influenciada pela relação inicial Ti/HAp, onde a maior quantidade de titânio inicial favorece a decomposição da HAp, e mostra algum efeito sobre o crescimento de grão da HAp, porém suas conseqüências sobre as propriedades mecânicas e biológicas do compósito não estão claramente compreendidas $[35,78]$.

\section{Biovidro}

Outra alternativa de reforço à HAp é a utilização de vidros bioativos (biovidro), visando melhorias tanto das propriedades mecânicas quanto da bioatividade da mesma [13, 33, 42, 52, 79, 80, 81]. Para reforço da HAp, destacase a utilização do biovidro, nome dado a uma série de composições de vidros baseados no sistema $\mathrm{Na}_{2} \mathrm{O}-\mathrm{CaO}$ $\mathrm{P}_{2} \mathrm{O}_{5}-\mathrm{SiO}_{2}$ que são conhecidos por serem particulados altamente bioativos [10, 24]. A melhoria das propriedades mecânicas do compósito HAp-vidro está relacionada principalmente à redução de porosidade, sendo diretamente dependente da temperatura de sinterização e da quantidade adicionada do vidro [52, 79-83]. 
Tabela III - Propriedades do compósito HAp-biovidro com dois óxidos [52].

[Table III - Properties of composite HAp-two oxides bioglass [52].]

\begin{tabular}{cccc}
\hline $\begin{array}{c}\text { Temperatura }\left({ }^{\circ} \mathrm{C}\right) \\
\text { biovidro }(\%)\end{array}$ & $\begin{array}{c}\text { Densidade } \\
\left(\mathrm{g} . \mathrm{cm}^{-3}\right)\end{array}$ & $\begin{array}{c}\text { Resistência à } \\
\text { compressão } \\
(\mathrm{MPa})\end{array}$ & $\begin{array}{c}\text { Dureza } \\
(\mathrm{HV})\end{array}$ \\
\hline $\begin{array}{c}1200{ }^{\circ} \mathrm{C} \\
5 \%\end{array}$ & $2,81 \pm 0,12$ & $64 \pm 27$ & $250 \pm 19$ \\
$1200{ }^{\circ} \mathrm{C}$ & $2,79 \pm 0,05$ & $67 \pm 17$ & $285 \pm 19$ \\
$10 \%$ & & & \\
$1300{ }^{\circ} \mathrm{C}$ & $2,77 \pm 0,05$ & $39 \pm 9$ & $238 \pm 26$ \\
$5 \%$ & & & \\
$1300{ }^{\circ} \mathrm{C}$ & $2,76 \pm 0,05$ & $53 \pm 11$ & $279 \pm 12$ \\
$10 \%$ & & & \\
\hline
\end{tabular}

Tabela IV - Propriedades do compósito HAp-biovidro com três óxidos [52].

[Table IV - Properties of HAp-three oxide bioglass composites [52].]

\begin{tabular}{cccc}
\hline $\begin{array}{c}\text { Temperatura }\left({ }^{\circ} \mathrm{C}\right) \\
\text { biovidro }(\%)\end{array}$ & $\begin{array}{c}\text { Densidade } \\
\left(\mathrm{g} . \mathrm{cm}^{-3}\right)\end{array}$ & $\begin{array}{c}\text { Resistência à } \\
\text { compressão } \\
(\mathrm{MPa})\end{array}$ & $\begin{array}{c}\text { Dureza } \\
(\mathrm{HV})\end{array}$ \\
\hline $\begin{array}{c}1200{ }^{\circ} \mathrm{C} \\
5 \%\end{array}$ & $2,79 \pm 0,05$ & $36 \pm 11$ & $222 \pm 23$ \\
$1200{ }^{\circ} \mathrm{C}$ & $2,76 \pm 0,11$ & $40 \pm 21$ & $259 \pm 15$ \\
$10 \%$ & & & \\
$1300{ }^{\circ} \mathrm{C}$ & $2,79 \pm 0,04$ & $55 \pm 14$ & $358 \pm 18$ \\
$5 \%$ & & & \\
$1300{ }^{\circ} \mathrm{C}$ & $2,79 \pm 0,14$ & $39 \pm 12$ & $301 \pm 8$ \\
$10 \%$ & & & \\
\hline
\end{tabular}

Oktar e Golle, em 2002, avaliaram a resistência à compressão, densidade e dureza do compósito HAp-biovidro com 2 óxidos (45,5 massa\% de $\mathrm{P}_{2} \mathrm{O}_{5}$ e 54,5 massa\% de CaO) ou com três óxidos (45 massa\% de $\mathrm{P}_{2} \mathrm{O}_{5}, 28$ massa\% de $\mathrm{CaO}$ e 27 massa\% de $\mathrm{Na}_{2} \mathrm{O}$ ). Foram adicionadas 5 e $10 \%$ de biovidro e utilizadas temperaturas de sinterização de $1200{ }^{\circ} \mathrm{C}$ e $1300^{\circ} \mathrm{C}$. Os autores observaram que a melhoria das propriedades avaliadas foi dependente de fatores como temperatura de sinterização e quantidade utilizada do reforço, conforme mostrado nas Tabelas III e IV [52].

A melhoria de propriedades como dureza e tenacidade à fratura foi observada por Lopes, Monteiro e Santos, em 1999 [81], no compósito HAp-vidro $\left(\mathrm{CaO}-\mathrm{P}_{2} \mathrm{O}_{5}\right)$. A melhoria da dureza foi associada com a redução de porosidade e a melhoria da tenacidade à fratura do compósito, que envolve um aumento da energia requerida para propagação de trincas, teve relação direta com a presença de outras fases como $\beta$-TCP, o que gera um aumento de volume criando forças de compressão em torno ponta da trinca [81].

O surgimento dessas fases depende da quantidade de vidro presente e da temperatura de sinterização utilizada e é resultado da reatividade entre HAp e a fase vítrea Para amostras sinterizadas em $1200{ }^{\circ} \mathrm{C}$, a fase $\beta$-TCP está sempre presente em compósitos de HAp reforçados por vidro e, se essa temperatura for de $1350{ }^{\circ} \mathrm{C}$, ocorre a presença adicional de pequena quantidade de $\alpha$-TCP na microestrutura. A redução da porosidade é esperada, já que se trata de um processso de sinterização com fase líquida, tendo também relação direta com a temperatura de sinterização utilizada, ou seja, o aumento da temperatura de sinterização leva a uma redução da porosidade presente, permitindo maior densificação [81]. Outra propriedade que também pode ser melhorada com a adição do biovidro $(32 \mathrm{~mol} \%$ de $\mathrm{CaO}, 23 \mathrm{~mol} \%$ de $\mathrm{Na}_{2} \mathrm{O}$ e $45 \mathrm{~mol} \% \mathrm{P}_{2} \mathrm{O}_{5}$ ) é a resistência à flexão, cujo valor também está diretamente relacionado com a temperatura de sinterização e teor de reforço usado, conforme observado em 2001 [80].

Quando 2,5 massa\% do biovidro foi adicionado, houve um aumento gradual na resistência à flexão com o aumento na temperatura de sinterização. Já na adição de 5\%, houve um aumento na resistência à flexão até a temperatura de sinterização de $1250{ }^{\circ} \mathrm{C}$, voltando a decrescer em temperaturas mais altas de sinterização. $\mathrm{O}$ aumento da resistência à flexão foi relacionado tanto à presença do vidro, que auxilia na remoção da porosidade da estrutura, quanto à decomposição da HAp em $\beta$-TCP, o que gera um aumento do volume, auxiliando nessa melhora. Em temperaturas mais altas, o $\beta$-TCP se transforma em $\alpha$-TCP, que em quantidades significativas resulta em um efeito prejudicial [80]. Resultados semelhantes foram encontrados em 1996, quando foi avaliado o efeito da adição do vidro (24 mol\% de $\mathrm{CaO}, 45 \mathrm{~mol} \% \mathrm{P}_{2} \mathrm{O}_{5}$ e $31 \mathrm{~mol} \% \mathrm{Na}_{2} \mathrm{O}$ ) sobre a resistência à flexão do compósito HAp-vidro [82]. Quando $2 \%$ de vidro foi adicionado e a temperatura de sinterização foi $1200{ }^{\circ} \mathrm{C}$,

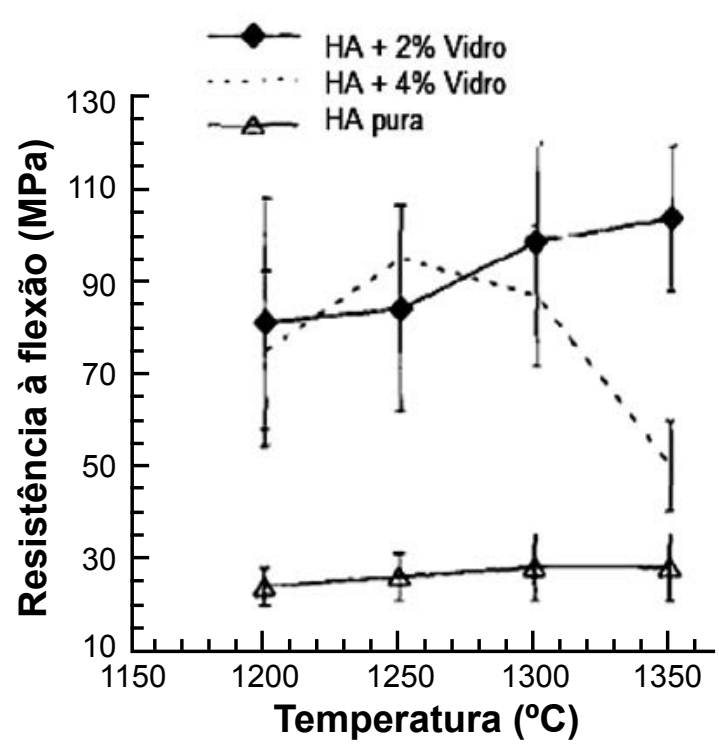

Figura 4: Efeito da temperatura de sinterização sobre a resistência à flexão da HAp, HAp-2\% vidro e HAp-4 peso\% vidro [82].

[Figure 4: Effect of the sintering temperature on flexural strength of HAp, HAp- 2wt\% glass and HA- 4 wt.\% glass [82].] 
$1250{ }^{\circ} \mathrm{C}$ ou $1300{ }^{\circ} \mathrm{C}$, foram encontrados a HAp e o $\beta$-TCP, enquanto que a $1350^{\circ} \mathrm{C}$ HAp e $\alpha$-TCP foram detectados. Já na adição de $4 \%$, verificou-se também a presença de $\beta$-TCP nas temperaturas de sinterização $1200{ }^{\circ} \mathrm{C}$ e $1250{ }^{\circ} \mathrm{C}$, porém em maiores quantidades a $1300{ }^{\circ} \mathrm{C}$ e $1350{ }^{\circ} \mathrm{C}$ coexistiram as fases HAp, $\alpha$-TCP e $\beta$-TCP. Em relação à resistência à flexão, para a adição de $2 \%$ de vidro, seu valor aumentou com o aumento da temperatura de sinterização. Quando foi adicionado $4 \%$ de vidro, a resistência teve seu valor elevado até $1300{ }^{\circ} \mathrm{C}$, quando começou a decrescer [82]. A relação encontrada entre a resistência à flexão e a temperatura de sinterização está apresentada na Fig. 4.

\section{Mulita}

Outro material investigado para utilização como reforço da HAp é a mulita $\left(3 \mathrm{Al}_{2} \mathrm{O}_{3} \cdot 2 \mathrm{SiO}_{2}\right)[47-49,84]$. Trata-se de um importante material cerâmico estrutural, resultado da reação entre alumina e sílica. Possui como principais características sua refratariedade, alta dureza e moderada tenacidade à fratura em relação à HAp [48]. Verifica-se que compósitos resultantes da incorporação de 10 massa\% de mulita após sinterização a $1350{ }^{\circ} \mathrm{C}$ por $2 \mathrm{~h}$ apresentaram como fases a HAp e o $\alpha$-TCP, além de mulita e de $\beta$-TCP; maiores adições de mulita induziram uma predominância de $\beta$-TCP, além de mulita, alumina, gehlenita $\left[\mathrm{Ca}_{2} \mathrm{Al}(\mathrm{SiAl}) \mathrm{O}_{7}\right]$, óxido de cálcio e HAp. A presença de mulita, dispersa na matriz de HAp, seria a responsável pela melhoria das propriedades mecânicas da HAp [48]. Já a presença de fases como $\mathrm{CaO}$ e gehlenita foram relacionadas à redução da dureza e módulo de elasticidade do compósito, independente de sua composição [47]. Propriedades como tenacidade à fratura, resistência à flexão e resistência à compressão foram medidas quando diferentes porcentagens de mulita foram adicionadas à HAp, onde se verificou que as maiores melhorias em tais propriedades foram obtidas quando se adicionou 20 ou $30 \%$ de mulita [49], conforme observado na Tabela V.

\section{Íons}

A adição de íons à HAp também vem sendo descrita com método para melhoria das propriedades mecânicas, microestrutura e biocompatibilidade da mesma. Íons de flúor, magnésio e de prata tem sido estudados [53-61].
O íon magnésio é um dos íons bivalentes mais importantes associados com apatitas biológicas. É conhecido por seu efeito significante em processos de mineralização. Sua incorporação à estrutura da HAp está associada a uma gradual transformação da HAp em $\beta$-TCP. Isso é explicado pelo fato do raio iônico do $\mathrm{Mg}^{2+}$ ser menor do que o raio iônico do íon $\mathrm{Ca}^{2+}$; essa substituição do íon maior pelo íon menor favorece a distorção da rede e conseqüentemente a formação de $\beta$-TCP [53-55]. A adição de $1 \%$ em massa de óxido de magnésio à HAp, quando sinterizada a $1250{ }^{\circ} \mathrm{C}$, favoreceu maior densificação, melhorou em $20 \%$ a dureza em relação ao valor da HAp pura e melhorou sua resistência à compressão em 33,8\% [56].

A adição do íon flúor à HAp foi também investigada e sua utilização é considerada como potencial alternativa para reparo de tecido ósseo devido à baixa solubilidade, boa compatibilidade e alta estabilidade térmica e química do material final produzido. Observou-se que a presença do flúor permitiu densificação superior a $90 \%$ na sinterização a $1250{ }^{\circ} \mathrm{C}$, além de limitação do crescimento de grão. Foi observada também significativo aumento da resistência à fratura em relação à hidroxiapatita pura, além de leve aumento do módulo de elasticidade e dureza [57].

A combinação de íon flúor com ítrio adicionada à HAp potencializou o efeito do flúor. A adição de flúor (2,5 mol\%) e de ítrio $\left(2,5,5\right.$ e 7,5 mol\%) à HAp, sinterizada a $1300{ }^{\circ} \mathrm{C}$ ocasionou um aumento da microdureza em relação à HAp pura. Esse aumento está relacionado, de forma direta, com a quantidade de dopagem. O maior valor de resistência à fratura foi obtido na sinterização a $1100{ }^{\circ} \mathrm{C}$, na dopagem de $2,5 \mathrm{~mol} \%$ de ítrio e $2,5 \mathrm{~mol} \%$ de flúor $\left(2,5 \% \mathrm{Y}^{3+}-2,5 \% \mathrm{~F}\right)$ [58].

Outro íon que vem sendo adicionado à HAp é a prata. Quando esse íon é adicionado, ocorre um aumento na atividade antimicrobiana contra microorganismos gram-positivos e gram-negativos [59-61]. Análises de difração de raios $X$ mostraram que não houve a formação de outra fase além da HAp, indicando que não ocorreu desestabilização ou dissolução da mesma, com a adição da prata [11, 62]. Outro estudo demonstrou a síntese da HAp com a incorporação do íon prata e observou a obtenção de satisfatórias propriedades tais como dureza e resistência, excelentes níveis de densificação, na sinterização a $1200{ }^{\circ} \mathrm{C}$ [63].

Tabela V - Propriedades mecânicas da hidroxiapatita pura e várias composições do compósito HAp- mulita [49].

[Table V - Mechanical properties of pure hydroxyapatite (HAp) and various HAp-mullite composites [49].]

\begin{tabular}{lcccc}
\hline \multicolumn{1}{c}{ Composição do pó } & $\begin{array}{c}\text { Condições de } \\
\text { sinterização } \\
{ }^{\circ} \mathrm{C} / \mathrm{h}\end{array}$ & $\begin{array}{c}\text { Tenacidade à } \\
\text { fratura } \\
\left(\mathrm{MPa}^{0.5}\right)\end{array}$ & $\begin{array}{c}\text { Resistência à } \\
\text { flexão } \\
(\mathrm{MPa})\end{array}$ & $\begin{array}{c}\text { Resistência à } \\
\text { compressão } \\
(\mathrm{MPa})\end{array}$ \\
\hline HAp pura & $1200 / 2$ & $0,6 \pm 0,01$ & - & $54,2 \pm 0,01$ \\
HAp- 10\% de mulita $(\mathrm{m} / \mathrm{m} \%)^{*}$ & $1400 / 2$ & $0,9 \pm 0,05$ & $51 \pm 1,2$ & $126,8 \pm 25,9$ \\
HAp- 20\% de mulita $(\mathrm{m} / \mathrm{m} \%)^{*}$ & $1350 / 2$ & $1,5 \pm 0,17$ & $80 \pm 3,5$ & $232,7 \pm 33,5$ \\
HAp- 30\% de mulita $(\mathrm{m} / \mathrm{m} \%)^{*}$ & $1350 / 2$ & $1,5 \pm 0,13$ & $75 \pm 2,7$ & $380,9 \pm 14,5$ \\
\hline
\end{tabular}

*Em relação à massa utilizada de HAp. 


\section{CONSIDERAÇÕES FINAIS}

A HAp é uma biocerâmica de uso clínico, possuindo várias indicações de uso na área biomédica, porém, devido às suas propriedades mecânicas, principalmente baixa resistência mecânica e alta fragilidade, sua utilização é restrita para uso em locais onde não são exigidos grandes esforços mecânicos. Uma alternativa para melhorar essas propriedades é a associação da HAp com outros materiais, visando unir as propriedades desejáveis dos materiais utilizados. A formação do compósito HAp-zircônia melhora as propriedades mecânicas da HAp, desde que controlada a sua decomposição, bem como a transformação de fase da zircônia. Isso é possível através da aplicação de processos de sinterização específicos utilizando temperaturas mais baixas e dentro de períodos menores de tempo ou utilização de aditivos tais como o óxido de cálcio $(\mathrm{CaO})$, óxido de magnésio $(\mathrm{MgO})$ e óxido de ítrio $\left(\mathrm{Y}_{2} \mathrm{O}_{3}\right)$ para gerar a zircônia parcialmente estabilizada (PSZ). A adição da alumina também apresenta resultados positivos para o compósito, porém as melhorias são limitadas devido a grande diferença de coeficiente de expansão térmica entre os dois materiais, além da ocorrência da degradação da HAp e reação da alumina, com a formação de fosfato de tricálcio e aluminatos de cálcio. A adição do titânio gera mudanças estruturais e processos de decomposição, com a presença de outras fases, tais como o dióxido de titânio e a perovskita $\left(\mathrm{CaTiO}_{3}\right)$, mesmo em técnicas que utilizam menores temperaturas no processamento, porém suas conseqüências sobre as propriedades mecânicas e biológicas do compósito não são claramente elucidadas na literatura. A utilização do vidro como segunda fase à HAp gera melhoria de propriedades como dureza e tenacidade à fratura, sendo essa melhoria principalmente associada à redução da porosidade, diretamente dependente da temperatura de sinterização e da quantidade adicionada do vidro. A adição de mulita em 20 ou 30\% gera melhoria de propriedades como resistência à fratura, resistência à flexão e resistência à compressão, porém a presença de fases como $\mathrm{CaO}$ e gehlenita estão relacionadas à redução da dureza e módulo de elasticidade do compósito, independente de sua composição. A adição de íons, tais como magnésio, flúor e ítrio, permite altas densificações e acarreta melhoria de propriedades como resistência à compressão, à fratura e dureza. Já a adição de íon de prata, além de melhorar dureza e resistência, ainda melhora a atividade antimicrobiana da HAp. A associação da HAp com outros materiais representa, na maioria dos casos, uma forma eficiente para melhoria das propriedades mecânicas, desde que sejam obedecidos critérios durante o processamento, tais como temperatura de sinterização e quantidade de reforço adicionada evitando assim efeitos deletérios sobre as propriedades do material final obtido.

\section{REFERÊNCIAS}

[1] M. A. Meyers, P. Chen, A. Y. Lin, Y. Seki, Progr. Mater. Sci. 53 (2008) 1-206.
[2] S. V. Dorozhkin, J. Mater. Sci. 42 (2007) 1061-1095.

[3] A. Yelten, S. Yilmaz, F. N. Oktar, Ceram. Int. 38 (2011) 2659-2665.

[4] Dj. Veljovic, I. Zalite, E. Palcevskis, I. Smiciklas, R. Petrovic, Dj. Janackovic, Ceram. Int. 36, 2 (2010) 595-603.

[5] M. Wang, Biomater. 24 (2003) 2133-2151.

[6] M. Vallet-Regi, J. M. Gonzalez-Calbet, Progr. Solid State Chem. 32 (2004) 1-31.

[7] S. J. Kalita, A. Bhardwaj, H. A. Bhatt, Mater. Sci. Eng. C 27 (2007) 441-449.

[8] L. L. Hench, J. Am. Ceram. Soc. 81 (1998)1705-1728.

[9] A. Farzadi, M. Solati-Hashjin, F. Bakhshi, Ceram. Int. 37 (2011) 65-71.

[10] S. M. Best, A. E. Porter, E. S. Thian, J. Huang, J. Eur. Ceram. Soc. 28 (2008) 1319-1327.

[11] R. Ramli, A. Z. O. Arawi, M. M. Mahat, A. F. Mohd, M. F. Zaman, R. Yahya, Int. Conf. Business, Eng. Ind. Appl. (2011) 180.

[12] C. S. Delfino, C. Ribeiro, G. F. Vieira,A.H.A.Bressiani, M. L. Turbino, Cerâmica 56, 340 (2010) 381-388.

[13] R. Ravarian, F. Moztarzadeh, M. Solati Hashjin, S. M. Rabiee, P. Khoshakhlagh, M. Tahriri, Ceram. Int. 36 (2010) 291-297.

[14] A. C. F. M. Costa, M. G. Lima, L. H. M. A. Lima, V. V. Cordeiro, K. M. S. Viana, C. V. Souza, H. L. Lira, Rev. Eletr. Mat. Proc. 4, 3 (2009) 29-38.

[15] C. Ergun, Ceram. Int. 37 (2011) 1143-1146.

[16] S. Ramesh, C. Y. Tan, W. H. Yeo, R. Tolouei, M. Amiriyan, I. Sopyan, W. D. Teng, Ceram. Int. 37 (2011) 599-606.

[17] K. A. Lacerda, F. S. Lameiras, V. V. Silva, Quim. Nova 32, 5 (2009) 1216-1221.

[18] M. S. Kim, Y. Kim, Mater. Lett. 66 (2011) 33-35.

[19] P. Y. Chen, A. Y. M. Lin, Y. S. Lin, Y. Seki, A. G. Stokes, J. Peyras, E. A. Olevsky, M. A. Meyers, J. McKittrick, J. Mech. Behavior Biomed. Mater. 1 (2008) 208-226.

[20] S. Ramesh, C. Y. Tan, R. Tolouei, M. Amiriyan, J. Purbolaksono, I. Sopyan, W. D. Teng, Mater. Des. 34 (2012) 148-154.

[21] S. Murakami, K. Kato, Y. Enari, M. Kamitakahara, N. Watanabe, K. Ioku, Ceram. Int. 38 (2012) 1649-1654.

[22] C. Chiu, H. Hsu, W. Tuan, Ceram. Int. 33 (2007) 715718

[23] I. Sopyan, S. Ramesh, N. A. Nawawi, A. Tampieri, S. Sprio, Ceram. Int. 37 (2011) 3703-3715.

[24] T. Kokubo, H. Kim, M. Kawashita, Biomater. 24 (2003) 2161-2175.

[25] I. Mobasherpour, M. S. Hashjin, S. S. R. Toosi, R. D. Kamachali, Ceram. Int. 35 (2009) 1569-1574.

[26] G. Muralithran, S. Ramesh, Ceram. Int. 26 (2000) 221230.

[27] S. Ramesh, C. Y. Tan, S. B. Bhaduri, W. D. Teng, Ceram. Int. 33 (2007) 1363-1367.

[28] S. Pramanik, A. K. Agarwal, K. N. Rai, A. Garg, Ceram. Int. 33 (2007) 419-426.

[29] L. Sun, C. C. Berndt, K. A. Gross, A. Kucuk, J. Biomed. Mater. Res. (Appl. Biomater.) 58 (2001) 570-592. 
[30] R. Z. Legeros, S. Lin, R. Rohanizadeh, D. Mijares, J. P. Legeros, J. Mater. Sci. Mater. Med. 14 (2003) 201-209. [31] K. A. Khor, Y. W. Gu, D. Pan, P. Cheang, Biomater. 25 (2004) 4009-4017.

[32] S. M. Kenny, M. Buggy, "Bone cements and fillers: A review", J. Mater. Sci. Mater. Med. 14 (2003) 923-928.

[33] M. M. Sebdani, M. H. Fathi, Ceram. Int. 38 (2012) 1325-1330.

[34] S. J. Kalita, D. Rokusek, S. Bose, H. L. Hosick, A. Bandyopadhyay, J. Biomed. Mater. Res. 71A (2004) 35-44. [35] C. Q. Ning, Y. Zhou, Biomater. 25 (2004) 3379-3387. [36] S. Salman, O. Gunduz, S. Yiumaz, M. L. Oveçoglu, R. L. Snyder, S. Agathopoulos, F. N. Oktar, Ceram. Int. 35 (2009) 2965-2971 .

[37] Z. Shen, E. Adolfsson, M. Nygren, L. Gao, H. Kawaoka, K. Niihara, Adv. Mater. 13, 3 (2001) 214-216.

[38] Y. Kong, C. Bae, S. Lee, H. Kim, H. Kim, Biomater. 26 (2005) 509- 517.

[39] Y. Kong, S. Kim, H. Kim. J. Am. Ceram. Soc. 82 (1999) 2963-2968.

[40] R. R. Rao, T. S. Kannan, Mater. Sci. Eng. C 20 (2002) 187-193.

[41] R. Kumar, K. H. Prakash, P. Cheang, K. A. Khor, Acta Mater. 53 (2005) 2327-2335.

[42] Y. Hu, X. Miao, Ceram. Int. 30 (2004) 1787-1791.

[43] V. V. Silva, F. S. Lameiras, R.Z. Domingues, Composites Sci. Technol. 61 (2001) 301-310.

[44] V. V. Silva, F. S. Lameiras, Mater. Charact. 45 (2000) 51-59.

[45] V. V. Silva, F. S. Lameiras, R. Z. Domingues, Ceram. Int. 27 (2001) 615-620.

[46] H. Ji, P. M. Marquis, Biomater. 13 (1992) 744-748.

[47] S. Nath, A. Dey, A. K. Mukhopadhyay, B. Basu, Mater. Sci. Eng. A 513-514 (2009) 197-201.

[48] S. Nath, K. Biswas, B. Basu, Scripta Mater. 58 (2008) 1054-1057.

[49] S. Nath, K. Biswas, K. Wang, R. K. Bordia, B. Basuw, J. Am. Ceram. Soc. 93 (2010) 1639-1649.

[50] P. A. Ramires, A. Romito, F. Cosentino, E. Milella, Biomater. 22 (2001) 1467-1474.

[51] R. R. Kumar, M. Wang, Mater. Lett. 55 (2002) 133-137. [52] F. N. Oktar, G. Goller, Ceram. Int. 28 (2002) 617-621. [53] I. V. Fadeev, L. I. Shvorneva, S. M. Barinov, V. P. Orlovskii, Inorg. Mater. 39 (2003) 947-950.

[54] E. Bertoni, A. Bigi, G. Cojazzi, M. Gandolfi, S. Panzavolta, N. Roveri, J. Inorg. Biochem. 72 (1998) 29-35. [55] A. Bigi, G. Falini, E. Foresti, M. Gazzano, A. Ripamonti, N. Roveri, J. Inorg. Biochem. 49 (1993) 69-78.

[56] S. J. Kalita, H. A. Bhatt, Mater. Sci. Eng. C 27 (2007) 837-848.

[57] A. Bianco, I. Cacciotti, M. Lombardi, L. Montanaro, E. Bemporad, M. Sebastiani, Ceram. Int. 36 (2010) 313-322.

[58] B. Basar, A. Tezcaner, D. Keskin, Z. Evis, Ceram. Int. 36 (2010) 1633-1643.
[59] M. Vukomanovic, I. Bracko, I. Poljansek, D. Uskokovic, S. D. Skapin, D. Suvorok, Cryst. Growth Des. 11 (2011) 3802-3812.

[60] T. N. Kim, Q. L. Feng, J. O. Kim, J. Wu, H. Wang, G. C. Chen, F. Z. Cui, J. Mater. Sci.: Mater. Med. 9 (1998) 129-134.

[61] Q. L. Feng, T. N. Kim, J. Wu, E. S. Park, J. O. Kim, D. Y. Lim, F. Z. Cui, Thin Solid Films 335 (1998) 214-219.

[62] M. Diaz,F. Barba, M. Miranda, F. Guitian, R. Torrecillas, J.S. Moyá, J. Nanomater. (2009), doi: 10.1155/2009/498505. [63] S. Nath, S. Kalmodia, B. Basu, J. Mater. Sci. Mater. Med. 21, 4 (2010) 1273-1287.

[64] C. J. Reidy, T. J. Fleming, S. Hampshire, M. R. Towler, Int. J. Appl, Ceram. Technol. 8 (2011) 1475-1485.

[65] D. J. Curran, T. J. Fleming, S. Hampshire, M. R. Towler, J. Mater. Sci. Mater. Med. 21, 4 (2010) 1109-1120.

[66] C. Piconi, G. Maccauro, Biomater. 20 (1999) 1-25.

[67] S. Salehi, M. H. Fathi, Ceram. Int. 36 (2010) 16591667.

[68] S. Nath, N. Sinha, B. Basu, Ceram. Int. 34 (2008) 15091520.

[69] S. Gautier, E. Champion, D. Bernache-Assollant, J. Eur. Ceram. Soc. 17 (1997) 1361-1369.

[70] S. Gautier, E. Champion, D. Bernache-Assollant, J. Mater. Sci. Mater. Med. 10 (1999) 533-540.

[71] S. Gautier, E. Champion, D. Bernache-Assollant, T. Chartier, J. Eur. Ceram. Soc. 19 (1999) 469-477.

[72] J. Choi, Y. Kong, H. Kim, J. Am. Ceram. Soc. 81 (1998) 1743-1748.

[73] S. Kim, H. Bang, J. Song, S. Park, Ceram. Int. 35 (2009) 1647-1650.

[74] J. Li, B. Fartash, L. Herrnansson, Biomater. 16 (1995) 417-422.

[75] E. Champion, S. Gautier, D. Bernache-Assollant, J. Mat. Sci. - Mater. Med. 7(1996) 125-130.

[76] K. Niespodziana, K. Jurczyk, J. Jakubowicz, M. Jurczyk, Mater. Chem. Phys. 123 (2010) 160-165.

[77] E. Milella, F. Cosentino, A. Licciulli, C. Massaro, Biomater. 22 (2001) 1425-1431.

[78] H. Li, K. A. Khora, P. Cheang, Biomater. 24 (2003) 949-957.

[79] J. D. Santos, J. C. Knowles, R. L. Reis, F. J. Monteiro, G. W. Hastings, Biomater. 15 (1994) 5-10.

[80] J. C. Knowles, S. Talal, J. D. Santos, Biomater. 17 (1996) 1437-1442.

[81] G. Georgiou, J. C. Knowles, Biomater. 22 (2001) 28112815.

[82] M. A. Lopes, F. J. Monteiro, J. D. Santos, Biomater. 20 (1999) 2085-2090.

[83] M. H. P. Silva, A. F. Lemos, I. R. Gibson, J. M. F. Ferreira, J. D. Santos, J. Non-Crystalline Solids 304 (2002) 286-292.

[84] T. Ebadzadeh, A. Behnamghader, R. Nemati, Ceram. Int. 37 (2011) 2887-2889.

(Rec. 27/11/2013, Rev. 17/04/2014, Ac.02/05/2014) 